\title{
Breast cancer diagnosis using spatial light interference microscopy
}

Hassaan Majeed

Mikhail E. Kandel

Kevin Han

Zelun Luo

Virgilia Macias

Krishnarao Tangella

Andre Balla

Gabriel Popescu

\section{SPIE.}




\title{
Breast cancer diagnosis using spatial light interference microscopy
}

\author{
Hassaan Majeed, ${ }^{\text {a }}$ Mikhail E. Kandel, ${ }^{a}$ Kevin Han, ${ }^{a}$ Zelun Luo, ${ }^{a}$ Virgilia Macias, ${ }^{b}$ Krishnarao Tangella, ${ }^{c}$ \\ Andre Balla,b and Gabriel Popescu $u^{a, k}$ \\ aUniversity of Illinois at Urbana-Champaign, Department of Electrical and Computer Engineering, Beckman Institute of \\ Advanced Science and Technology, Quantitative Light Imaging Laboratory, 405 North Mathews Avenue, Urbana, Illinois 61801, United States \\ bUniversity of Illinois at Chicago, Department of Pathology, 840 South Wood Street, Suite 130 CSN, Chicago, Illinois 60612, United States \\ 'University of Illinois at Urbana-Champaign, Christie Clinic and Department of Pathology, 1400 West Park Street, \\ Urbana, Illinois 61801, United States
}

\begin{abstract}
The standard practice in histopathology of breast cancers is to examine a hematoxylin and eosin (H\&E) stained tissue biopsy under a microscope to diagnose whether a lesion is benign or malignant. This determination is made based on a manual, qualitative inspection, making it subject to investigator bias and resulting in low throughput. Hence, a quantitative, label-free, and high-throughput diagnosis method is highly desirable. We present here preliminary results showing the potential of quantitative phase imaging for breast cancer screening and help with differential diagnosis. We generated phase maps of unstained breast tissue biopsies using spatial light interference microscopy (SLIM). As a first step toward quantitative diagnosis based on SLIM, we carried out a qualitative evaluation of our label-free images. These images were shown to two pathologists who classified each case as either benign or malignant. This diagnosis was then compared against the diagnosis of the two pathologists on corresponding H\&E stained tissue images and the number of agreements were counted. The agreement between SLIM and H\&E based diagnosis was $88 \%$ for the first pathologist and $87 \%$ for the second. Our results demonstrate the potential and promise of SLIM for quantitative, label-free, and high-throughput diagnosis. @ 2015 Society of Photo-Optical Instrumentation Engineers (SPIE) [DOI: 10.1117/1.JBO.20.11.111210]
\end{abstract}

Keywords: breast cancer; histopathology; quantitative phase imaging; microscopy; label-free imaging; spatial light interference microscopy.

Paper 150163SSPRR received Mar. 15, 2015; accepted for publication Jul. 17, 2015; published online Aug. $20,2015$.

\section{Introduction}

Breast cancer is the second most common form of cancer diagnosed worldwide, accounting for $11.9 \%$ of all cancers diagnosed in 2012. ${ }^{1}$ In spite of the high incidence and burden of the disease, the current histopathological analysis used for the diagnosis of breast cancers suffers from certain shortcomings. When an abnormality in the breast is discovered during a screening procedure such as mammography, a tissue biopsy is obtained by the pathologist and the section of tissue is stained using hematoxylin and eosin (H\&E). This staining provides the necessary contrast needed for investigation of key morphological features by a trained, board certified pathologist using a conventional bright-field microscope. Since this investigation is qualitative and relies on the subjective perception of the investigator, it leads to both intra- and interobserver discrepancy. ${ }^{2-4}$ Furthermore, the investigation is manual and the process does not easily lend itself to automation. This is, in part, due to the fact that machine learning classifiers that are built for automated image analysis require consistency in the values of image parameters that are the basis for classification. In bright-field imaging of stained tissue, both variations in staining intensity and system illumination can lead to changes in pixel values that are unrelated to the morphology of tissue. This complicates the development of high-throughput automated image analysis

*Address all correspondence to: Gabriel Popescu, E-mail: gpopescu@illinois .edu systems, meaning that the standard diagnosis method still relies on manual investigation and, as a result, suffers from low throughput. This can, in some cases, result in late disease diagnosis-a critical shortcoming given that early diagnosis significantly improves chances of survival. . $^{5,6}$

Quantitative phase imaging (QPI) refers to a subset of labelfree microscopy techniques where contrast in the image is generated by the variation of optical path length across the sample. ${ }^{7}$ The resulting image is a phase map $\phi(x, y)$ that is a quantitative measure of the product of the difference between the refractive index of the tissue, $n_{t}$, and that of its surrounding medium, $n_{m}$, and the thickness of the tissue, $t$, given by the equation

$\phi(x, y)=\frac{2 \pi}{\lambda}\left[n_{t}(x, y)-n_{m}\right] t(x, y)$,

where $\lambda$ is the wavelength of light. ${ }^{7-16}$ Since the information in the phase image $\phi(x, y)$ is a quantitative measure of the morphology of the tissue biopsy, diagnosis based on QPI provides the potential to eliminate inter- and intraobserver variation. Futhermore, building classifiers for automated analysis is also simpler since the pixel values in the phase image represent a physical property of the tissue that will not vary unless tissue morphology changes-providing consistency in parameters that may be used as a basis for classification.

$1083-3668 / 2015 / \$ 25.00$ @ 2015 SPIE 
Spatial light interference microscopy (SLIM) provides access to this phase information by measuring four interferograms formed by the scattered and unscattered fields and solving for $\phi$. As shown in Fig. 1, the SLIM module is constructed as an add-on to a commercial phase contrast microscope (Zeiss Axio Z1 Observer). The module consists of a 4-f system comprising lenses L1 and L2 that relay the conjugate image plane outside of the microscope onto the CCD camera (Andor, Zyla). At the Fourier plane of the first lens L1, the scattered and unscattered fields are separated owing to the fact that this plane is conjugate to the microscope pupil plane. A spatial light modulator (SLM), manufactured by Meadowlark Optics, is placed at this Fourier plane, and by generating patterns on the SLM, the phase difference between the scattered and unscattered light is set at four different values to obtain four different interferrograms at the CCD plane. As was shown by Wang et al., the four interferograms can then be solved to obtain $\phi .^{17}$

It has been shown in previous publications from our group that SLIM provides diffraction-limited resolution as well as low spatial and temporal noise leading to optical path length sensitivity of less than a nanometer. ${ }^{17-20}$ The utility of refractive index of tissue (accessible through the phase images obtained by SLIM) as a marker for prostate tissue malignancy has also been shown in previous publications, which motivates the current application of SLIM as a histopathological analysis technique for breast tissue biopsies. ${ }^{21,22}$

In this work, we present preliminary results that show the potential of a SLIM based technique for diagnosis of breast cancers. Specifically, the resolution and contrast of SLIM phase images for diagnostic purposes were evaluated qualitatively by two board certified pathologists. As outlined in detail in the following sections, using the standard $\mathrm{H} \& \mathrm{E}$ staining

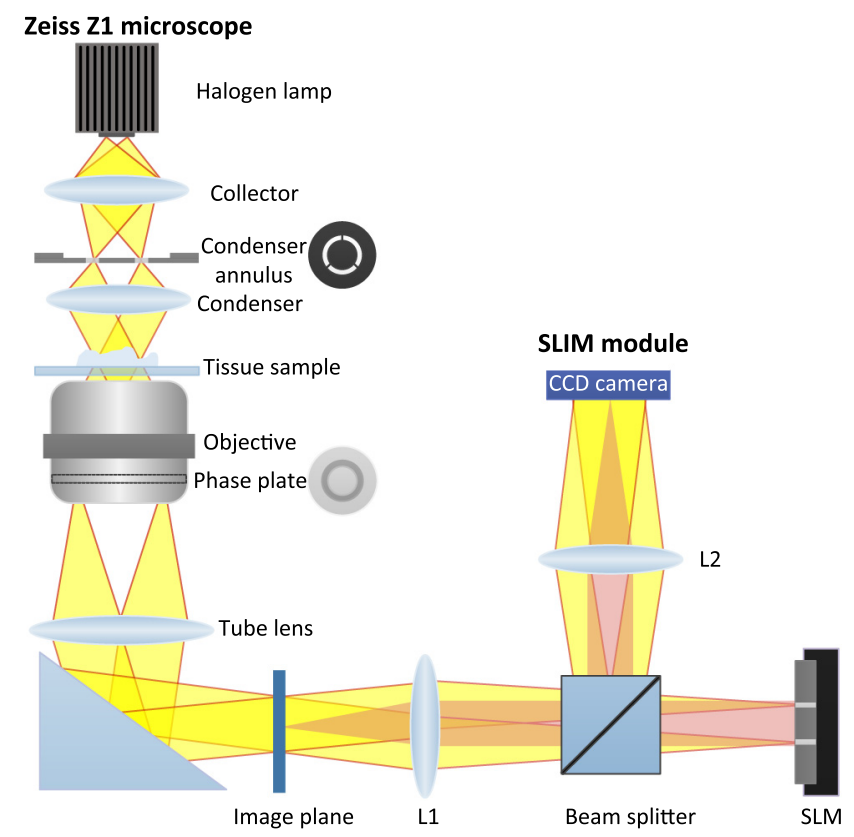

Fig. 1 Spatial light interference microscopy (SLIM) optical setup. The phase is retrieved by shifting the phase of the scattered light with respect to that of the unscattered light by using a spatial light modulator (SLM) at the Fourier plane of lens L1. Four interferograms, correponding to four different SLM phase shifts, are obtained and used to compute the phase. ${ }^{17}$ based diagnosis protocol as a benchmark, the success of the pathologists in carrying out diagnosis on SLIM images was measured. Our results provide an indication of the signal-tonoise ratio available to us for subsequent quantitative analyses for carrying out diagnosis based on the relative phase values of various tissue components.

\section{Experimental Procedures}

\subsection{Tissue Microarray}

The samples comprised a tissue microarray (TMA) of cores constructed from breast tissue biopsies of 400 different patients. Each biopsy was formalin fixed and paraffin embedded before sectioning it into slices of $4 \mu \mathrm{m}$ thickness each using a microtome. Two parallel, adjacent sections were selected from each biopsy and one of these sections was stained using H\&E, leaving the other one unstained. Cores were then constructed for both the stained and unstained tissue, and these were mounted on separate slides after de-paraffinization, using xylene as the mounting medium. The stained samples were imaged using a bright-field microscope, and their images served as a reference for evaluating diagnosis on the unstained samples using SLIM. The slides were obtained from our collaborating pathologist, Dr. Andre Balla, at the University of Illinois at Chicago. The procedures used in this study for conducting experiments using human subjects were approved by the institute review board at the University of Illinois at Urbana-Champaign (IRB Protocol Number 13900).

\subsection{Slide Scanning and Mosaicking}

The TMA was imaged using our SLIM imaging system (Fig. 1), equipped with a $40 x / 0.75$ NA phase contrast objective. A slide scanning software, developed in-house in Visual $\mathrm{C}++$, was used to obtain the raw images for the entire microscope slide (scanning area $\sim 20 \mathrm{~mm} \times 45 \mathrm{~mm})$ at high throughput $(\sim 2 \mathrm{~h}$ per slide).

The scanning speed of our SLIM imaging system can be assessed by comparing it with that of a commercial slide scanner. For example, the Zeiss Axio Scan.Z1 slide scanner is able to scan a $15 \times 15 \mathrm{~mm}^{2}$ area, at a $0.22 \mu \mathrm{m} /$ pixel sampling rate, in $240 \mathrm{~s}$. The SLIM system scans the same area at $0.125 \mu \mathrm{m} /$ pixel in $1638 \mathrm{~s}$. Scanning at the same resolution as the Zeiss instrument would improve the speed of the SLIM system by a factor of $(0.22 / 0.125)^{2}=3.1$, to $528 \mathrm{~s}$. Thus, our SLIM scanner is only a factor of 2.2 slower than the commercial Zeiss scanner, which is remarkable, especially considering that we record four intensity images for each SLIM image.

The phase maps were extracted from the acquired intensity images using a MATLAB ${ }^{\circ}$-based code. A C++-based code was used for stitching the mosaic for the entire slide and segmenting out each individual core for subsequent processing and analysis. As shown in Fig. 2, our processing allows the visualization of the entire TMA from the slide scale to the subcellular scale within each core. The phase image of one entire TMA slide used in this study has been uploaded on a server, which can be accessed from Ref. 23 and viewed at several zoom levels. As illustrated in Fig. 2(c), our label-free SLIM images clearly delineate the epithelial stromal boundary allowing for assessment of tumor malignancy. 


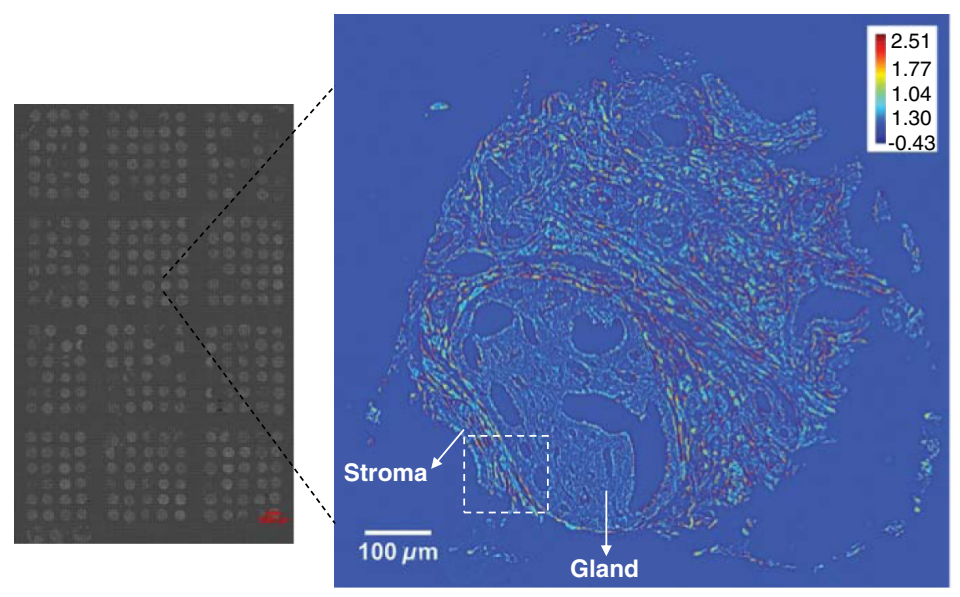

(a) (b)

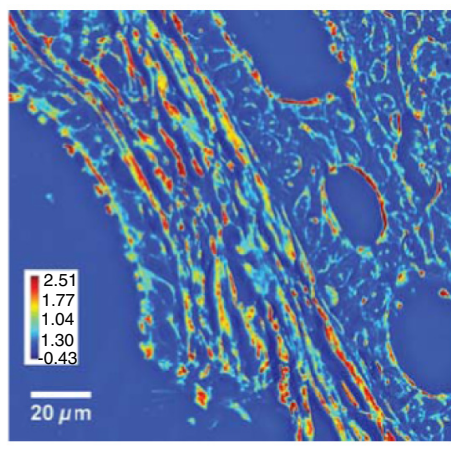

(c)

Colorbar: phase values in radians

Fig. 2 (a) The SLIM image of an entire tissue microarray (TMA) slide $(20 \mathrm{~mm} \times 45 \mathrm{~mm}$ ) scanned and stitched using software developed in-house, (b) label-free SLIM image of a single TMA core delineating the boundary between the tumor and its extracellular environment, and (c) magnified image of region indicated in (b) clearly showing tumor cell nuclei and collagen fibers, specific to epithelial and stromal regions.

\subsection{Pathologist Training Procedure}

In order to assess the diagnostic capabilities of our SLIM imaging modality, we asked two board certified pathologists to evaluate our SLIM images. For this preliminary study, we selected 109 cores for evaluation, and stacks of both SLIM and corresponding H\&E images for these cores were assembled in ImageJ. Since pathologists are generally trained to recognize morphological features in H\&E stained tissue, we performed a training step before the actual test. In this training step, out of the total cohort of 109 cores, 10 benign and 10 malignant cores (classified as such by a third board certified pathologist $a$ priori)

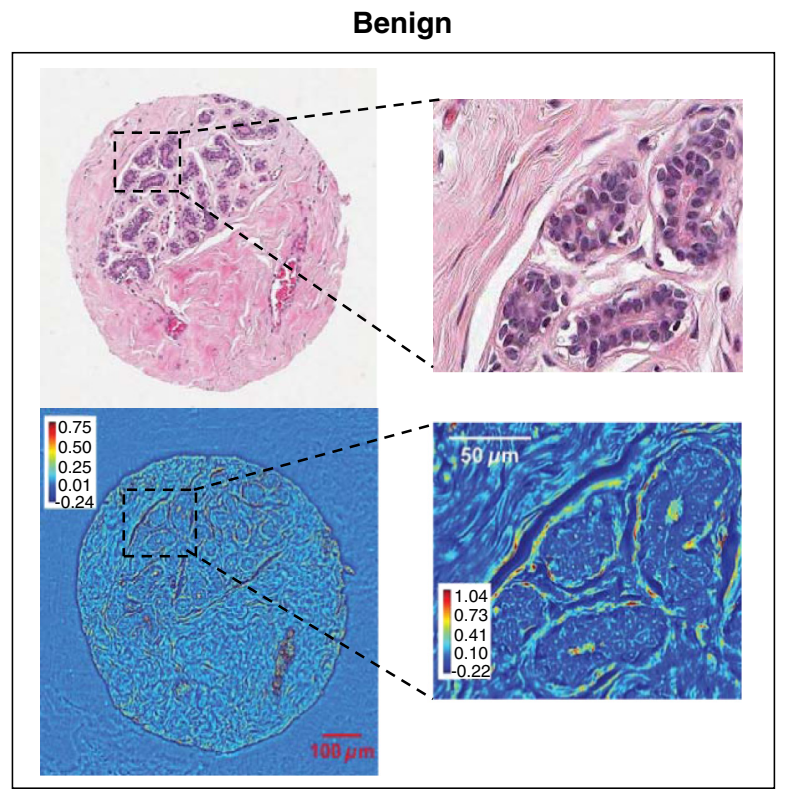

(a) were chosen and their SLIM and H\&E images were shown side by side to each pathologist. By comparing the SLIM and H\&E images for each core, the pathologists were able to learn how to interpret the tissue morphological details from SLIM phase maps. The total training time for each pathologist ranged from 10 to $15 \mathrm{~min}$, approximately.

Figure 3 compares and contrasts how different tissue components are resolved in SLIM and H\&E stained tissue images. Due to the fact that our system uses phase contrast illumination, SLIM images inherit some of the halo artifact that is characteristic of phase contrast images. This effect is due to the fact that

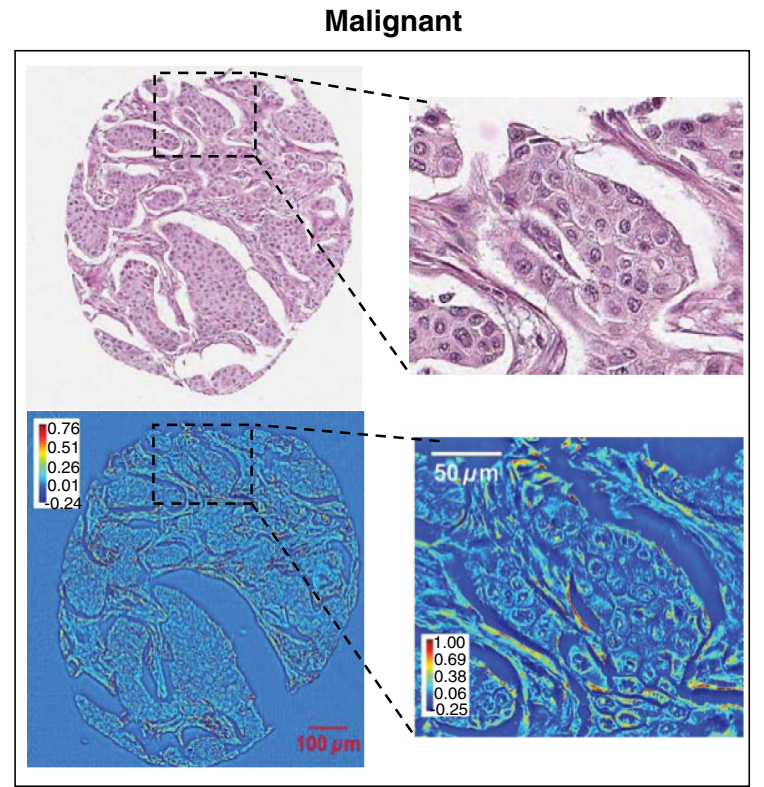

(b)

Fig. 3 Comparison between H\&E stained bright-field microscopy (top row) and SLIM (bottom row) images in their respective abilities to resolve tissue morphology for (a) benign and (b) malignant cases. The H\&E images were obtained from stained sections that were adjacent to the unstained sections used for SLIM imaging. Color bars are in radians. 
some of the high spatial frequency components pass through the low spatial frequency region (ring) in the Fourier plane. As a result, negative phase values are observed at sharp edges in the SLIM phase images. Since the study presented here is based on visual interpretation of SLIM images by pathologists, the halo artifact does not affect our results because, as illustrated in Fig. 3, the tissue morphology is not obscured by the presence of the halo artifact. Typically, for highly quantitative studies based on measuring physical parameters, we use halo removal algorithms developed in-house to recover halo-free quantitative phase images. ${ }^{24-26}$

\subsection{Pathologist Diagnosis Using SLIM}

After the completion of the training step, at the testing stage, each pathologist was first shown the stack of SLIM images for all of the 109 cores chosen. The pathologist classified each core as either benign or malignant. The process was repeated for

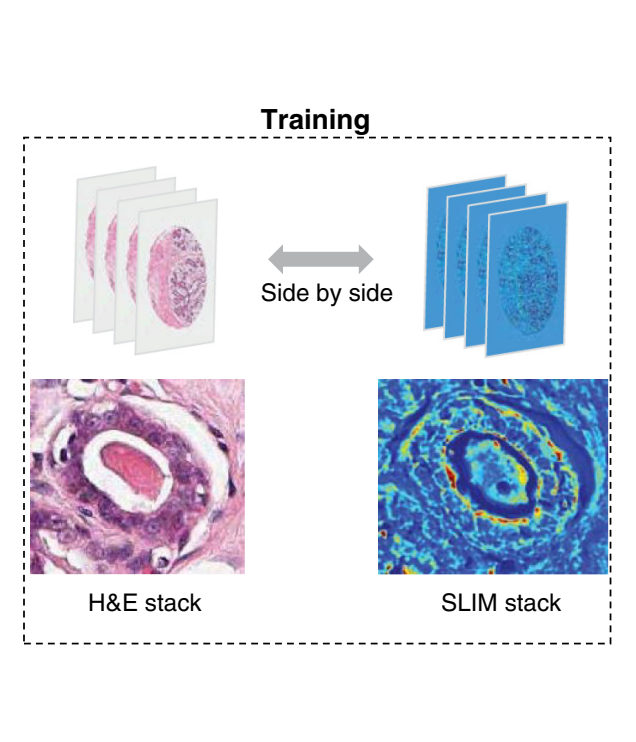

(a)

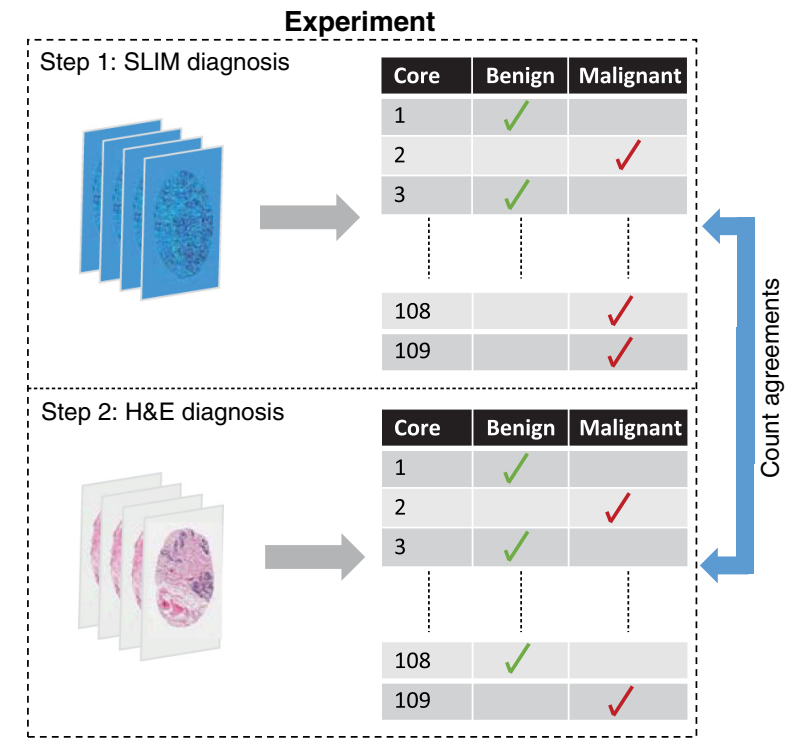

(b)

Fig. 4 Diagnosis using SLIM images by pathologists and comparison with H\&E based diagnosis. (a) The training step was performed prior to the experiment. For 20 cores out of the cohort of 109 cores in total, both SLIM and H\&E images were shown side by side to each pathologist, training the pathologist to interpret morphology from SLIM images by comparing features with corresponding H\&E images. (b) For the entire 109-core cohort, each pathologist classified a core as either benign or malignant by looking at their SLIM images. The process was repeated for H\&E images, and number of agreements between the two diagnoses were counted for each pathologist.

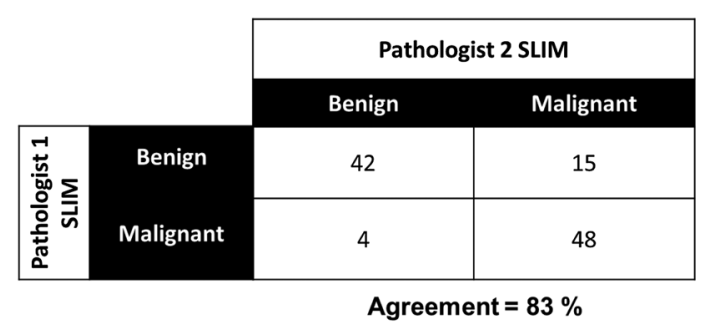

(a)

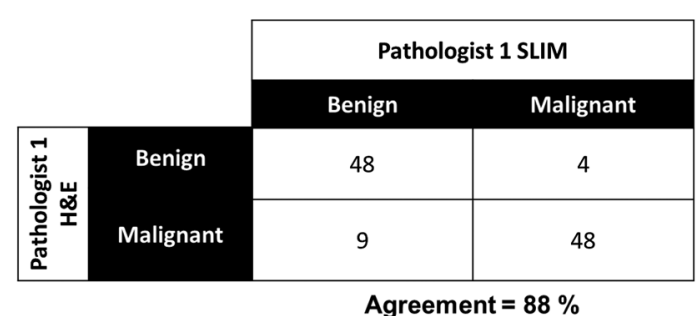

(c)

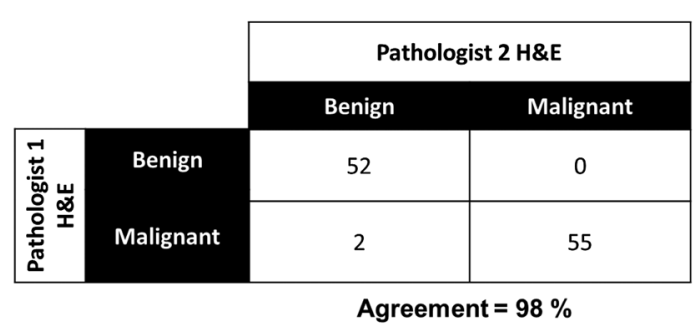

(b)

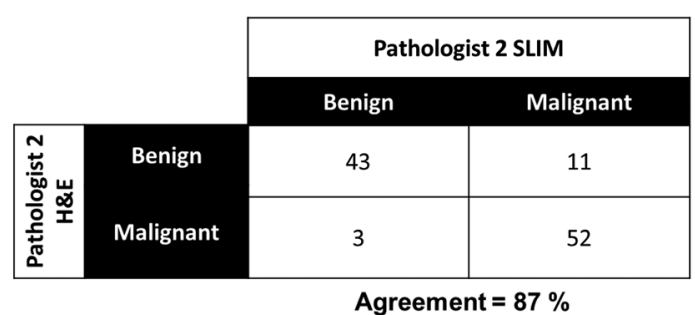

(d)

Fig. 5 Confusion matrices showing results of qualitative diagnosis carried out by two pathologists on both SLIM and H\&E stained tissue images for 109 cores. (a) Pathologist agreement on SLIM images and (b) pathologist agreement on H\&E images. (c) and (d) Agreement between ratings on SLIM and H\&E images for each pathologist. 
the stack of H\&E images for the same 109 cores. Using each pathologist's diagnosis on the H\&E stained cores as the gold standard, the success of diagnosis using SLIM images was measured by counting the number of agreements between SLIM and H\&E based diagnoses. The entire exercise is schematically depicted in Fig. 4.

\section{Results}

The results of the core classification carried out by the two pathologists on both SLIM and H\&E images are summarized in Fig. 5. As shown in Figs. 5(c) and 5(d), the success rate of diagnosis on SLIM images (considering diagnosis on H\&E as the gold standard) for pathologist 1 was $88 \%$ and that for pathologist 2 was 87\%. As shown in Figs. 5(a) and 5(b), the agreement between the two pathologists when rating SLIM images stood at $83 \%$, whereas the same for H\&E images was much higher at $98 \%$. The lower agreement between the two pathologists on SLIM images is not surprising when one takes into account the fact that, as part of their professional training, pathologists are trained to interpret images of H\&E stained tissue for a number of years, whereas, for this experiment, the training time for SLIM images was only a few minutes. We expect the agreement between the diagnoses of the two pathologists on SLIM images to increase significantly with longer training in interpreting SLIM images.

\section{Summary and Future Work}

Our preliminary results show the capability of our label-free imaging modality in resolving morphological features relevant for diagnosis of breast cancer. While this qualitative analysis shows the potential of QPI for diagnosing malignancy in breast cancer, the long-term aim is to address the shortcomings in conventional histopathology with regards to inter- and intraobserver variability and low throughput by searching for quantitative parameters for classifying benign and malignant tumors. As has been reported in literature before, the simultaneous measurement of both the amplitude and phase of the optical imaging field associated with tissues can be used for extracting scattering parameters, such as mean-scattering length and tissue anisotropy parameter, that can be used to characterize the cellular scale organization of tissue. ${ }^{27-29}$ Since malignancy is associated with changes in tissue organization and morphological state of cells, comparing scattering parameters between benign and malignant tumors has the potential to provide a quantitative basis for diagnosis. Hence, our future work is focused on leveraging the quantitative information regarding tissue morphology available to us in phase maps to come up with parameters for classifying benign and malignant tumors, and building software platforms that can achieve this in an automated fashion.

In addition to discovering quantitative bases for diagnosis, we also hope to use our imaging modality for discovering new prognostic biomarkers. There is a search in the scientific community for new, readily accessible biomarkers to serve as prognostic indicators for breast cancer as the current set of biomarkers (histological grade, tumor size, hormonal receptor status, etc.) are insufficient in predicting outcomes for some patients. ${ }^{30}$ We hope to address this need by discovering new biomarkers, using our label-free images to serve as predictors of patient outcome.

\section{Acknowledgments}

This work was supported by National Science Foundation (Grant CBET-1040462 MRI), Phi Optics, and Agilent Technologies. For more information, visit http://light.ece.illinois.edu/.

\section{References}

1. International Agency for Research on Cancer, "Latest world cancer statistics. Global cancer burden rises to 14.1 million new cases in 2012: marked increase in breast cancers must be addressed," 12 December 2013, http://www.iarc.fr/en/media-centre/pr/2013/pdfs/pr223_E.pdf (10 March 2015).

2. A. Benard et al., "Infrared imaging in breast cancer: automated tissue component recognition and spectral characterization of breast cancer cells as well as the tumor microenvironment," Analyst 139(5), 1044 1056 (2014).

3. E. Rakha et al., "Breast cancer prognostic classification in the molecular era: the role of histological grade," Breast Cancer Res. 12(4), 207 (2010).

4. R. K. Jain et al., "Atypical ductal hyperplasia: interobserver and intraobserver variability," Mod. Pathol. 24(7), 917-923 (2011).

5. M. J. Walsh et al., "Attenuated total reflectance Fourier-transform infrared spectroscopic imaging for breast histopathology," Vib. Spectrosc. 60(0), 23-28 (2012).

6. American Cancer Society, Breast Cancer Facts \& Figures 2011-2012, American Cancer Society, Inc., Atlanta (2015).

7. G. Popescu, Quantitative Phase Imaging of Cells and Tissues, McGraw Hill, New York (2011).

8. C. Mann et al., "High-resolution quantitative phase-contrast microscopy by digital holography," Opt. Express 13(22), 8693-8698 (2005).

9. M. Kim, Digital Holographic Microscopy: Principles, Techniques, and Applications, Springer, New York (2011).

10. X. Ou et al., "Quantitative phase imaging via Fourier ptychographic microscopy," Opt. Lett. 38(22), 4845-4848 (2013).

11. J. Wu et al., "Harmonically matched grating-based full-field quantitative high-resolution phase microscope for observing dynamics of transparent biological samples," Opt. Express 15(26), 18141-18155 (2007).

12. P. Memmolo et al., "3D morphometry of red blood cells by digital holography," Cytometry 85(12), 1030-1036 (2014).

13. P. Memmolo et al., "Twin-beams digital holography for $3 \mathrm{D}$ tracking and quantitative phase-contrast microscopy in microfluidics," Opt. Express 19(25), 25833-25842 (2011).

14. M. Paturzo et al., "Microscopy imaging and quantitative phase contrast mapping in turbid microfluidic channels by digital holography," $L a b$ Chip 12(17), 3073-3076 (2012).

15. P. Ferraro et al., "Quantitative phase microscopy of microstructures with extended measurement range and correction of chromatic aberrations by multiwavelength digital holography," Opt. Express 15(22), 1459114600 (2007).

16. Y. Park et al., "Diffraction phase and fluorescence microscopy," Opt. Express 14(18), 8263-8268 (2006).

17. Z. Wang et al., "Spatial light interference microscopy (SLIM)," Opt. Express 19(2), 1016-1026 (2011).

18. T. Kim et al., "White-light diffraction tomography of unlabelled live cells," Nat. Photonics 8(3), 256-263 (2014).

19. Z. Wang et al., "Label-free intracellular transport measured by spatial light interference microscopy," J. Biomed. Opt. 16(2), 026019 (2011).

20. Z. Wang et al., "Topography and refractometry of nanostructures using spatial light interference microscopy," Opt. Lett. 35(2), 208-210 (2010).

21. Z. Wang et al., "Tissue refractive index as marker of disease," J. Biomed. Opt. 16(11), 116017 (2011).

22. S. Sridharan et al., "Prediction of prostate cancer recurrence using quantitative phase imaging," Sci. Rep. 5, 9976 (2015).

23. M. Kandel, H. Kevin, and Z. Luo, "CATMAID v0.24 projects: breast," July 2014, http://128.174.217.193/ (10 March 2015).

24. T. H. Nguyen et al., "Quantitative phase imaging with partially coherent illumination," Opt. Lett. 39(19), 5511-5514 (2014).

25. C. Edwards et al., "Effects of spatial coherence in diffraction phase microscopy,” Opt. Express 22(5), 5133-5146 (2014).

26. T. Nguyen et al., (in preparation). 
27. Z. Wang, H. Ding, and G. Popescu, "Scattering-phase theorem," Opt. Lett. 36(7), 1215-1217 (2011).

28. H. Ding et al., "Fourier transform light scattering of inhomogeneous and dynamic structures," Phys. Rev. Lett. 101(23), 238102 (2008).

29. H. Ding et al., "Optical properties of tissues quantified by Fouriertransform light scattering," Opt. Lett. 34(9), 1372-1374 (2009).

30. M. W. Conklin et al., "Aligned collagen is a prognostic signature for survival in human breast carcinoma," Am. J. Pathol. 178(3), 12211232 (2011).

Hassaan Majeed is a PhD candidate in the Bioengineering Department at the University of Illinois at Urbana-Champaign. Working in the Quantitative Light Imaging (QLI) Lab at the Beckman Institute of Advanced Science and Technology, his research is focused on using quantitative phase imaging for developing new methods for breast cancer diagnosis as well as looking for indicators of prognosis.

Mikhail E. Kandel is currently pursuing his master's degree in electrical engineering at the University of Illinois at Urbana-Champaign. Working in the QLI Lab at the Beckman Institute, his thesis work has been focused on developing software tools for high-throughput microscopy.

Kevin Han is a PhD student in the Electrical Engineering and Computer Sciences Department at the University of California, Berkeley. He completed his undergraduate degree in electrical engineering at the University of Illinois at Urbana-Champaign. His research interests include nanophotonics, optoelectronics, and novel two-dimensional materials.

Zelun Luo is currently a master's student in computer science at Stanford University. He finished his bachelor's degree in computer engineering at the University of Illinois at Urbana-Champaign, during which he worked in the QLI Laboratory, developing the Image Stitching and Core Segmentation software for Tissue Microarray scans (available at https://github.com/dingnigefei/superstitchous2 .0). His main interests include computer vision and bioimaging.

Virgilia Macias is a research assistant professor in the Department of Pathology at University of Illinois at Chicago. She is a pathologist with background in oncologic surgical pathology. As coinvestigator, she has performed translational research under several $\mathrm{NIH}$-funded grants. She has been focused on the application of high-throughput technologies, such as tissue microarray construction, laser capture microdissection, and digital imaging for the analysis of molecular markers in prostate, colon, and breast cancer.

Krishnarao Tangella is a clinical pathologist and medical director of Laboratory at the Presence Covenant Medical Center in Urbana, Illinois. He is also a clinical assistant professor in the Departments of Pathology and Internal Medicine at the University of Illinois, College of Medicine. His research interests include cellular biomedical imaging and optical characteristics of cancers.

Andre Balla is professor and director of transdisciplinary pathology at the University of Illinois at Chicago. His clinical focus is anatomic pathology with special interest in gynecologic pathology and prostate cancer. His research interests are in the areas of tissue banking, tissue microarrays, application of molecular techniques to tissue pathology, prostate cancer clinical outcomes prediction methods, and the effect of environmental agents on prostate cancer progression and metastasis.

Gabriel Popescu is an associate professor in electrical and computer engineering at University of Illinois at Urbana-Champaign. His research is focused in biomedical optics, with special emphasis on interferometric label-free imaging. He has published a book on QPI, authored 110 journal publications, 120 conference presentations, 24 patents, and gave 120 invited/keynote/plenary talks. He founded Phi Optics, Inc., a startup company that commercializes quantitative phase imaging technology. He is a fellow of OSA and SPIE. 